\title{
CONOCIMIENTO Y CREENCIAS SOBRE EL CONDÓN MASCULINO EN LOS ESTUDIANTES DE LA UNIVERSIDAD NACIONAL DEL CENTRO DEL PERÚ
}

\author{
KNOWLEDGE AND BELIEFS ABOUT THE MALE CONDOM IN STUDENTS OF UNIVERSIDAD \\ NACIONAL DEL CENTRO DEL PERU
}

\author{
Dra. Layli Maravi Baldeón, Dr. Rigoberto Zúñiga Mera, Mg. Soledad Garay Quintana \\ Instituto de Investigación de la Facultad de Trabajo social
}

\section{RESUMEN}

Diversos y recientes estudios indican que la información acerca de la importancia del uso del preservativo en la prevención de las ITS y el SIDA es bastante conocida y aceptada. Sin embargo se conoce también por los mismos estudios que esta información no se refleja necesariamente en el uso del condón masculino en sus prácticas sexuales, esto se debe a que subsisten aun muchos mitos y prejuicios en su utilización y a la información distorsionada obtenida de fuentes poco serias. Nuestra localidad no está exenta de esta problemática por lo cual se hace necesario conocer que información manejan sobre el condón masculino, así mismo sobre las creencias relacionadas al condón masculino. El problema de la investigación fue ¿Cuál es el nivel de conocimiento y que creencias tienen los estudiantes de la UNCP sobre el condón masculino?, objetivo general: determinar el nivel de conocimiento y las creencias que tienen los estudiantes de la UNCP sobre el condón masculino, y la hipótesis: los estudiantes de la UNCP poseen un nivel de conocimiento insuficiente y creencias desfavorables acerca del condón masculino. En el estudio fue descriptivo transversal, las técnicas que se utilizaron fueron la encuesta estructurada y el grupo focal. La población estuvo constituida por los estudiantes de todas las facultades de la UNCP siendo un numero de 8753 y la muestra de 406 estudiantes. Las conclusiones a las que arribamos son 1) Los alumnos de la UNCP tienen conocimientos suficientes sobre el condón, 2) los alumnos de la UNCP tienen creencias desfavorables acerca del condón masculino.

Palabras clave: Condón, conocimiento, creencia.

Diverse and recent studies indicate that the information brings over of the importance of the use of the condom (preservative) in the prevention of the ITS and the AIDS is known enough and accepted. Nevertheless it is known also by the same studies that this information is not reflected necessarily in the use of the masculine condom in his sexual practices, this owes to that many myths and prejudices survive even in itd utilization and to the distorted information obtained of undignified sources (fountains). Our locality is not exempt from this problematic for which it becomes necessary to know that they handle information about the masculine condom, likewise on the beliefs related to the masculine condom. The problem of the investigation was which is the level of knowledge and which beliefs the students of the UNCP have about the masculine condom?, general aim: To determine the level of knowledge and the beliefs that UNCP students have on the masculine condom, and the hypothesis: The students of the UNCP possess a level of insufficient knowledge and the beliefs that have are unfavorable about masculine condom. The study was descriptive, transversely, the technologies that were in use were the structured survey and the focal group. The population was constituted by the students of all the faculties of the UNCP being a number of 8753 and the sample of 406 students. The conclusions at which we arrived were 1) The pupils of the UNCP have sufficient knowledge on the condom, 2) the pupils of the UNCP have unfavorable beliefs of the masculine condom.

Key words: Condom, knowledge, belief. 


\section{INTRODUCCIÓN}

La investigación "Conocimiento y creencias sobre el condón masculino" ha sido realizada con los jóvenes universitarios de la UNCP, población que puede considerarse de alto riesgo, las medidas recomendables para evitar o disminuir el riesgo de exposición a ITSs incluyendo el VIH son: abstinencia sexual, relación monógama, relación sexual protegida.

Las prácticas sexuales se han considerado sin riesgo (sexo seguro) cuando el intercambio de secreciones como sangre, semen y fluidos vaginales es nulo y se utiliza el condón.

La medida más eficaz dentro de las prácticas de poco riesgo es la utilización del condón masculino que implica factores sociales como personales. Dentro de los factores personales está lo referido a los conocimientos y las creencias que influyen significativamente, ya que los conocimientos involucran datos correctos, en este caso, acerca del condón, y las creencias representan las ideas, pensamientos acerca del condón que pueden contribuir a prevenir un daño a la salud o aumentar su posibilidad.

Por estas razones consideramos que es imperativo identificar las creencias y los conocimientos que tienen los alumnos de la UNCP

El problema de la investigación fue ¿cuál es el nivel de conocimiento y que creencias tienen los estudiantes de la UNCP sobre el condón masculino? El objetivo general: determinar el nivel de conocimiento y las creencias que tienen los estudiantes de la UNCP sobre el condón masculino, y la hipótesis: los estudiantes de la UNCP poseen un nivel de conocimiento insuficiente y creencias desfavorables acerca del condón masculino.

El estudio fue descriptivo transversal y las técnicas que se utilizaron fueron la encuesta estructurada y el grupo focal.

a población estuvo constituida por los estudiantes de todas las facultades de la UNCP en un número de 8753 y la muestra de 406 estudiantes.

\section{MATERIAL Y MÉTODO}

\section{METODOLOGÍA DEL ESTUDIO}

NIVEL DE INVESTIGACIÓN

Básico, el estudio fue descriptivo transversal.

\section{MÉTODO}

El método utilizado fue el de análisis y síntesis que permitió tener un conocimiento sobre el tema elaborado de manera particular para explicarlo a nivel general y viceversa.

TÉCNICAS: Se utilizará:

1) La encuesta estructurada.

2) Grupal focal.

\section{DISEÑO METODOLÓGICO}

\section{POBLACIÓN Y MUESTRA}

LAPOBLACIÓN está constituida por los estudiantes de todas las Facultades de la Universidad Nacional del Centro del Perú que son un número de 8,753.

MUESTRA: 406 estudiantes

\section{RESULTADOS Y DISCUSIÓN}

El recojo de la información de la presente investigación se realizó a través de dos técnicas

1. La encuesta, mediante un cuestionario con 24 preguntas, de las cuales 15 correspondían a la variable conocimientos y 9 preguntas la variable creencias.

2. Grupo focal, que se trabajó en un primer momento con 15 jóvenes de las diferentes facultades de las UNCP, de ambos sexos, siete varones y ocho mujeres; de edad comprendidas entre 18 y 23 años, seleccionados al azar a través de dos informantes claves. En un segundo momento, se separó el grupo por sexo, a fin de profundizar y facilitar la interacción en este tema que toca la intimidad de los participantes. Con ambos grupos se realizó dos sesiones. Las sesiones fueron 
conducidas por las autoras, que actuaron como moderadoras.

HIPÓTESIS ESPECÍFICA No 1: los estudiantes de la UNCP poseen un nivel de conocimientos insuficientes acerca del condón masculino.

La variable conocimientos tuvo 15 preguntas en el cuestionario referidos a los siguientes indicadores; definición (1), función que cumple (1), importancia (3), utilización (10). Para el análisis de los resultados se consideró las respuestas correctas como conocimientos suficientes, considerando que el alumno posee los conocimientos adecuados sobre el condón masculino y, las respuestas incorrectas como conocimientos insuficientes, referido a que los alumnos de la UNCP no tienen conocimientos adecuados sobre el condón masculino.

Se encontró que en cuatro preguntas los conocimientos fueron insuficientes.

1) Referente a la función principal que cumple el condón masculino el $57.38 \%$ responde que el condón previene el embarazo y previene el cáncer, el $42.62 \%$ si responde que la función principal del condón es evitar el intercambio de fluidos corporales.

2) Referente a la pregunta si utiliza el condón masculino en sus relaciones coitales, el $59.85 \%$ responde que en algunas ocasiones y nunca, y el $40.15 \%$ responde que siempre.

3) La pregunta si en la primera relación sexual utilizó el condón masculino, el $65.02 \%$ responde que no lo utilizo, y el $34.98 \%$ responde que si utilizo.

4) Respecto a la afirmación si para usar el condón masculino no se necesita un adiestramiento previo, el $48.77 \%$ responde que es falso lo que es correcto, el $51.23 \%$ responde que la afirmación es verdadera, lo que es incorrecto.

Las otras once preguntas que corresponde a la variable de conocimientos fueron respondidas de manera suficiente. Consideramos que, a pesar que la mayoría de las preguntas fueron respondidas correctamente, los conocimientos que tienen los estudiantes sobre el condón masculino está lejos de identificar al condón como dispositivo de prevención, su preocupación sexual va por otro lado, como es su satisfacción.
El condón debe utilizarse siempre, un adagio muy cierto frente al tema dice: no importa con quien, cual es la preferencia sexual o la de la pareja ni cuantas veces se haga sino como se hace el acto sexual y en ese cómo; lo importante es utilizar el condón. No existen grupos de riesgo lo que existe son conductas de riesgo.

"Un factor de riesgo es una característica 0 circunstancia detectable en individuos o en grupos asociados con una probabilidad incrementada de experimentar un daño a la salud"

En este caso la falta de conocimientos suficientes conducirá a una conducta de riesgo.

De otro lado señalamos que nuestros resultados coinciden con la investigación realizada en Colombia sobre la prevalencia del uso del condón masculino en la primera relación sexual que fue de $68.6 \%$

Objetivo especifico $\mathrm{N}^{0}$ 1: determinar el nivel de conocimientos que poseen los estudiantes de la UNCP sobre el condón masculino.

Como se explicó, los conocimientos que poseen los estudiantes de la UNCP sobre el condón masculino son suficientes porque de las 15 preguntas, once han sido respondidas correctamente y 4 de manera incorrecta.

\begin{tabular}{|c|c|c|c|c|}
\hline Definición & $\begin{array}{c}\text { İtem } \\
1\end{array}$ & $\begin{array}{c}\text { Suficiente } \\
364\end{array}$ & $\begin{array}{c}\text { Insuficiente } \\
42\end{array}$ & $\begin{array}{c}\text { TOTAL } \\
406\end{array}$ \\
\hline $\begin{array}{l}\text { Función } \\
\text { cumple }\end{array}$ & 0 & 173 & 233 & 406 \\
\hline Importancia & 3 & 343 & 63 & 406 \\
\hline Importancia & 4 & 163 & 243 & 406 \\
\hline Importancia & 5 & 339 & 67 & 406 \\
\hline Utilización & 6 & 313 & 93 & 406 \\
\hline Utilización & 7 & 360 & 46 & 406 \\
\hline Utilización & 8 & 334 & 72 & 406 \\
\hline Utilización & 9 & 142 & 264 & 406 \\
\hline Utilización & 10 & 238 & 168 & 406 \\
\hline Utilización & 11 & 198 & 208 & 406 \\
\hline Utilización & 12 & 336 & 70 & 406 \\
\hline Utilización & 13 & 337 & 69 & 406 \\
\hline Utilización & 14 & 367 & 39 & 406 \\
\hline Utilización & 15 & 369 & 37 & 406 \\
\hline
\end{tabular}

Referente a la hipótesis específica № 1 , se plantea:

Hipótesis nula.- Los estudiantes de la UNCP poseen un nivel de conocimientos insuficientes a cerca del condón masculino. 
Hipótesis alterna.- Los estudiantes de la UNCP poseen un nivel de conocimientos suficientes acerca del condón masculino.

\begin{tabular}{ccc}
\hline & $\begin{array}{c}\text { Conocimiento } \\
\text { suficiente }\end{array}$ & $\begin{array}{c}\text { Conocimiento } \\
\text { Insuficiente }\end{array}$ \\
\hline Promedio & 291.73 & 114.27 \\
\hline
\end{tabular}

Como el promedio de conocimientos suficiente, es mayor al promedio de conocimientos insuficiente, se rechaza la hipótesis nula y se concluye que el nivel de conocimientos en los alumnos de la UNCP es suficiente.

Referente al grupo focal, como se explicó, en un primer momento se trabajó con 15 jóvenes de ambos sexos, pero al desarrollar la dinámica se observo que la comunicación debido al tema no era el más adecuado, los varones y las mujeres evitaban dar sus opiniones abiertamente, por ello se optó por separar al grupo por sexo, y se trabajó con las siguiente pregunta.

\section{1. ¿Con quien usa el condón?}

Los hombres opinaron que lo usan para protegerse sobre todo de una enfermedad y de un embarazo. Las mujeres opinaron que aceptarían el uso para prevenir un embarazo, no mostraron preocupación frente al contagio de una enfermedad porque "no tienen relaciones con un desconocido"

"En primer lugar, si es un vacilón, lo uso para no infectarme y para que no me vayan a embarcar".

Los varones realizan una división en las mujeres: las enamoradas, novias y las de encuentros casuales.

"Si es con tu enamorada es para que no salga embarazada, pero si es un vacilón, es para evitar infecciones"

Las mujeres manifestaron que usarían el condón solo si sus parejas lo proponen, ninguna aceptó la idea de que ellas podrían proponer su uso.

"No podría decir a mi enamorado ponte el condón porque pensaría que soy una jugadora".

"Si en la primera relación me propone usar el condón sentiría que el desconfía de mi"

Se observa nuevamente la división que los varones realizan entre pareja formal y la ocasional.
"Depende con quien lo haga si es con la firme, nunca; si es con las otras, si uso el condón"

Se nota la confianza en la pareja formal, por lo que no necesitan protegerse contra enfermedades, con la presunción de la fidelidad.

El varón siente que es fiel a su enamorada, porque usa condón con las parejas ocasionales.

Las mujeres manifiestan que usarían el condón con su pareja para prevenir un embarazo.

"El condón es fácil de usarlo y no te causa daño como las pastillas".

Las mujeres no hacen una división como los varones; para ellas no hay parejas formales y parejas ocasionales. Podemos concluir que los conocimientos no son aplicados, que el peligro de enfermar por no usar el condón es algo que no se percibe con claridad, sobre todo en las mujeres, es más bien un riesgo que se encuentra en los otros.

Los varones también tienen una baja percepción de riesgo, asociado a pareja formal y fidelidad, sigue asociando hombría con mayor número de parejas, conquistas como antesala de conductas de riesgo. La separación que realizan de los tipos de mujeres, hace que su percepción de peligro se encuentre distorsionada. La encuesta da como resultado que tienen conocimientos suficientes pero, se observa, que estos no son utilizados en su comportamiento cotidiano.

HIPÓTESIS ESPECÍFICA No 2: los estudiantes de la UNCP tienen creencias desfavorables a cerca del condón masculino. La variable creencias tuvo 9 preguntas en la cuestionario referidas a los siguientes indicadores: Sensación: opinión sobre propuesta de uso, calificativo a mujer y hombre que proponen su uso, decisión de uso y no uso. Razones por qué no se debe usar el condón, eficacia del condón.

Para el análisis de los resultados se consideró las respuestas incorrectas como creencias desfavorables aquellos que son dañinos y contribuyen para un comportamiento de riesgo.

Se encontró de las nueve preguntas, tres que corresponde a creencias desfavorables. 
1. El condón masculino contribuye a la sequedad vaginal, $38.42 \%$, limita el acto sexual $40.15 \%$, limita el ritmo $21.43 \%$, respuestas incorrectas totalizando el $100 \%$, la respuesta correcta fue ninguna de la anteriores que tuvo $0 \%$.

2. Opinión sobre propuesta de usar condón. El $8.62 \%$ responde porque no hay sentimientos, el $53.94 \%$ porque hay desconfianza y el $37.43 \%$ porque tiene varias parejas, respuestas incorrectas, totalizando el $100 \%$. La respuesta correcta es ninguna de las anteriores que tuvo $0 \%$.

3. Razones por lo que no se deberían usar el condón, el $31.77 \%$ responde que disminuye el placer sexual, el $12.81 \%$ responde porque causa inflamación y el $44.21 \%$ responde causa incomodidad en la relación. La respuesta correcta es ninguna de las anteriores. Que tuvo $0 \%$.

Las otras 6 preguntas fueron respondidas de manera favorable.

Se observa que persisten creencias alrededor del condón; como, que no se siente igual, que incomoda 0 interfiere el erotismo de la relación sexual, a esto se suma la creencia que deben usar el condón masculino los que son infieles y los que tienen parejas ocasionales; también se observa que las relaciones sexuales entre varones y mujeres están marcados por el machismo, donde la iniciativa sexual, el riesgo y la aventura es masculina y la sumisión, la entrega total las pocas habilidades comunicativas para llegar a acuerdos de protección, siguen siendo características femeninas.

Consideramos que las creencias están basadas en el temor de ver cuestionada la virilidad porque interfiere con el acto sexual o peor aún, atenta contra su desempeño sexual, al poner a prueba su firmeza eréctil, y para evitar situaciones embarazosas prefieren descalificarlo de antemano con afirmaciones de que limita el ritmo, contribuyen a la sequedad vaginal, causa inflamación, etc.

También se aprecia que son los varones los que deciden cuando y bajo qué circunstancias se ponen el condón debido a la necesidad de mantener permanentemente el control del ejercicio del acto sexual.
Según Moser: "Las relaciones de género se definen como aquellas relaciones socialmente construidas entre varones y mujeres, son relaciones de poder con acceso diferenciado a recursos y toma de decisiones".

Vargas, Imelda, señalan que genero es la relación entre los sexos es con primacía, hegemonía y predominio masculino.

Nuestros resultados coinciden con el trabajo efectuado por Adriana Martínez Ramírez, Martha Villa, quienes señalaron que las creencias que tienen los adolescentes, escolares sobre el condón masculino, son desfavorables.

Objetivo especifico $N^{0} 2$ : identificar las creencias que tiene los estudiantes de la UNCP sobre el condón masculino.

Como se explicó, las creencias que poseen los estudiantes de la UNCP sobre el condón masculino son desfavorables

\section{Creencias para no usar el condón}

2. Creencias sobre la decisión del uso.

\begin{tabular}{|c|c|c|c|c|}
\hline & İtem & Favorable & Desfavorable & TOTAL \\
\hline Sensación & 1 & 243 & 163 & 406 \\
\hline Sensación & 2 & 0 & 406 & 406 \\
\hline Opinión propuesta uso & 3 & 0 & 406 & 406 \\
\hline $\begin{array}{l}\text { Calificativo a mujer que } \\
\text { propone el uso }\end{array}$ & 4 & 362 & 44 & 406 \\
\hline $\begin{array}{l}\text { Calificativo a hombre que } \\
\text { propone el uso }\end{array}$ & 5 & 224 & 182 & 406 \\
\hline Decisión de uso & 6 & 352 & 54 & 406 \\
\hline $\begin{array}{l}\text { Razones por la que no se } \\
\text { debe usar }\end{array}$ & 7 & 0 & 406 & 406 \\
\hline Eficacia del condón & 8 & 342 & 64 & 406 \\
\hline Usó condón & 9 & 297 & 109 & 406 \\
\hline
\end{tabular}

Referente a la hipótesis especifica $\mathrm{N}^{0} 2$ :

Hipótesis nula.- los estudiantes de la UNCP tienen creencias desfavorables acerca del condón masculino.

Hipótesis alterna.- los estudiantes de la UNCP tienen creencias favorables acerca del condón masculino.

\begin{tabular}{ccc}
\hline & $\begin{array}{c}\text { Creencias } \\
\text { favorables }\end{array}$ & $\begin{array}{c}\text { Creencias } \\
\text { desfavorables }\end{array}$ \\
\hline Promedio & 202.22 & 203.78 \\
\hline
\end{tabular}

Como el promedio de creencias desfavorables es mayor al promedio de creencias favorables, 
se acepta la hipótesis nula y se concluye que los estudiantes de la UNCP tienen creencias desfavorables acerca del condón masculino.

Referente al grupo focal se trabajó la variable creencias con las siguientes preguntas.

- ¿Quién debe proponer el uso del condón masculino?

- ¿Cuáles son las razones por las que no se debe usar el condón masculino?

Los hombres manifestaron que no usarían el condón porque quita la sensibilidad

"No es igual tener relaciones con el preservativo"

También manifestaron que no lo usarían por temor al rechazo de parte de la mujer.

"Algunas se enojan lo toman como una ofensa dicen yo no ando con cualquiera"

No usar condón puede ser sinónimo de amor, porque es una demostración de confianza.

También manifestaron que no usarían el condón cuando se les presenta la oportunidad y no podrían desperdiciarla.

"No podría quedar mal; frente a una oportunidad, hay que cumplir"

\section{¿Quién se detiene por no tener condón?}

Los jóvenes se presentan como conquistadores incapaces de desechar una oportunidad cumpliendo su deber como machos

"El hombre llega hasta donde la mujer quiere"

Las mujeres refirieron que no usarían el condón porque no se atreven a exigir a su pareja por el temor a que puedan pensar de ella, ser tildadas de lanzadas.

"Yo confió en mi pareja"

"Yo no me meto con cualquiera"

Otra creencia es que los condones son delgados pueden romperse y también a la falta de habilidad para colocárselo. Varones y mujeres enfatizaron mediante afirmaciones que quita la sensibilidad.

"No es lo mismo definitivamente es diferente el roce"
"Es como si acariciaras con guantes".

"Se pierde el encanto"

"Estas en acción y haces un alto".

El condón se percibe como una barrera entre la pareja y el placer, la connotación de lo natural está muy arraigada antes que la idea de prevención.

Inclusive su uso se ve como algo dañino a la salud y a la relación, reduce el placer, es una interferencia artificial frente al desenvolvimiento natural de la relación sexual.

¿Quién debe proponer el uso del condón masculino?

La mayoría de los varones estuvieron de acuerdo en afirmar que son ellos los que proponen el uso del condón, a pesar que se puede generar un conflicto.

"La mujer dice: ¿Quién te imaginas que soy?

"Si la mujer me propone usarlo desconfiaría de ella"

Algunos de los participantes mencionaron que llevan un condón, de tal manera estar preparados para cualquier eventualidad.

Las mujeres afirman que proponer el uso del condón es algo de mal gusto, aceptan su poca capacidad de diálogo y negociación en este tema por temor a ser descalificadas, asimismo reconocen que la decisión debe ser tomada por la pareja.

Se aprecia que el condón masculino puede contribuir a reforzar la imagen de dominio, llevar un condón reafirma la hombría y da prestigio dentro del grupo. Se podría decir que llevan un condón, más para presumir que como medida preventiva.

Referente a la mujer se observa que perciben dos modelos de mujer: la buena inocente sin malicia, y la mala, jugadora. No se aprecia el factor responsabilidad ni el empoderamiento en este tema.

La investigación cuantitativa refleja que los alumnos de la UNCP tienen conocimientos suficientes y creencias desfavorables, sin embargo en el trabajo cualitativo se aprecia que los conocimientos suficientes se ven opacados por las creencias y esto se podría explicar con la teoría de la DISONANCIA 
COGNOSCITIVA que ayuda a comprender porque la adquisición de conocimientos, aunque estos sean buenos o muy buenos, no implican necesariamente un cambio de comportamiento, esta teoría desarrollada por LEON FESTINGE en 1957, plantea que no siempre se actúa de acuerdo a lo que se sabe. Podría decirse que una persona está en disonancia siempre que realiza una acción para la cual tiene información que considerada en sí misma, conduce a evitar dicha acción.

\section{CONCLUSIONES}

- El nivel de conocimientos sobre el condón masculino que poseen los estudiantes de la UNCP son suficientes.

- Las creencias que tienen los estudiantes de la UNCP sobre el condón masculino son desfavorables.

\section{REFERENCIAS BIBLIOGRAFÍCAS}

1. Alarcón B. I. Comportamiento Sexual en varones adolescentes de colegios estatales de Lima, Cusco e Iquitos. Tesis (Maestría en Psicología) Universidad Peruana Cayetano Heredia, Lima 1992.

2. Cacéres C. Rosasco AM, Muñoz S. Gotuzzo E. Mandel J. Hearst N. Necesidades en relación con la sexualidad humana y el SIDA entre estudiantes y profesores de escuela secundaría en Lima Rev. Lat.Psicol 1992;24:109-122.

3. Ceballos Ospino, Guillermo Augusto Psic, Adalberto Campo-Arias, MD. "Prevalencia de uso de condón en la primera relación sexual en adolescentes de santa marta, Colombia: diferencias por género", 2005.

4. Gayet, Cecilia M en D, Juárez, Fatima PhD, Pedrosa/Laura A. M en C, Magis, Carlos M en SP, "Uso del condón entre adolescentes mexicanos para la prevención de las infecciones de transmisión sexual", 2000.

5. Martínez Ramírez, Adriana Florencia, Martha Villaseñor Farías y Alfredo Celis de la Rosa "El condón masculino y su eficacia. información y creencias en adolescentes escolares". 